\title{
RESPIRE: A Spectral Kurtosis-based Method to Extract Respiration Rate from Wearable PPG Signals
}

\author{
Harishchandra Dubey*, Nicholas Constant ${ }^{\dagger}$, Kunal Mankodiya ${ }^{\dagger}$ \\ *Center for Robust Speech Systems, University of Texas at Dallas, Richardson, TX-75080, USA \\ ${ }^{\dagger}$ Wearable Biosensing Lab, University of Rhode Island, Kingston, RI-02881, USA \\ dubey@ele.uri.edu, kunalm@uri.edu
}

\begin{abstract}
In this paper, we present the design of a wearable photoplethysmography (PPG) system, R-band for acquiring the PPG signals. PPG signals are influenced by the respiration or breathing process and hence can be used for estimation of respiration rate. R-Band detects the PPG signal that is routed to a Bluetooth low energy device such as a nearbyplaced smartphone via microprocessor. Further, we developed an algorithm based on Extreme Learning Machine (ELM) regression for the estimation of respiration rate. We proposed spectral kurtosis features that are fused with the state-ofthe-art respiratory-induced amplitude, intensity and frequency variations-based features for the estimation of respiration rate (in units of breaths per minute). In contrast to the neural network (NN), ELM does not require tuning of hidden layer parameter and thus drastically reduces the computational cost as compared to NN trained by the standard backpropagation algorithm. We evaluated the proposed algorithm on Capnobase data available in the public domain.
\end{abstract}

Keywords-Wearable; Photoplethysmography; Spectral Kurtosis; Extreme Learning Machine (ELM) Regression; Respiration Rate; Cardiovascular Diseases (CVD).

\section{INTRODUCTION}

Cardiovascular Diseases (CVDs) accounted for $32 \%$ of deaths in USA for the year 2010 [1]. Heart diseases are among major cause of deaths in US [2]. Respiratory disorders related to sleep increase the risk of stroke often referred as sleep apnea disorders. The respiratory disorders were found to increase the risk of stroke [3]. Thus, the variations in the respiration rates needed to be monitored. It demands for wearable systems [4]-[6] powered with algorithms for accurate estimation of respiration rate. Respiration disorders related with sleep is known to degrade the development and growth in children. Breathing disorders lead to depression and anxiety etc.. Authors in [7] studied the prevalence of anxiety and depression in patients with chronic breathing disorders. The respiration or breathing rate is an important criterion for diagnosis of respiratory illness such as Pneumonia in children [8]. The present paper provided a solution to this problem by designing and developing a prototype of a wearable PPG system named R-band (for Respiration-band). Further, we proposed an algorithm that accurately estimates the respiration rate using spectral kurtosis features fused with the state-of-the-art features. Fused feature set is used to train an Extreme Learning Machine (ELM) regression

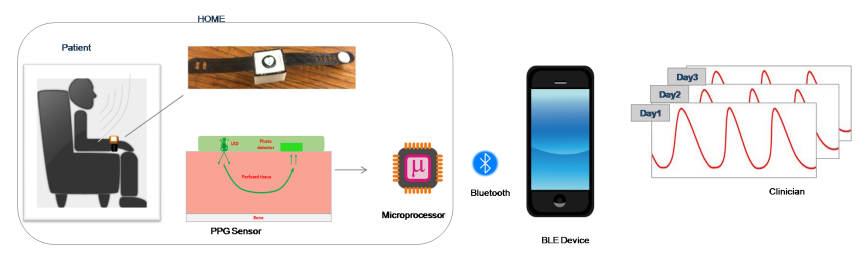

Figure 1. Conceptual view of proposed framework. PPG signals from RBand wristband were routed through a microprocessor into a nearby-placed Bluetooth device such as smartphone. Spectral kurtosis-based method extracts the respiration rate from PPG signal.

model. The algorithm was benchmarked on the standard Capnobase dataset. Figure 1 shows the concept of proposed framework.

II. BACKGROUND \& RELATED WORKS

Remote monitoring of respiration or breathing rate can lead to taking suggestive prevention before the disorders become profound. Using wearables such as PPG wrist-band lead to an inexpensive tool for monitoring the respiration rate and hence tracking the related health problems in a timely and continuous manner [9], [10]. Authors in [11] developed a method for getting a reliable estimates of breathing rates in children using mobile devices. The RR estimates obtained by the clinicians had been unreliable and inaccurate for preliminary diagnosis [12] in some instances. The reflectiontype photoplethysmography (PPG) technology detects the changes in intensity of light upon reflection from the skin. PPG signal is related to the respiration rhythm and hence can be used to estimate the respiration rate (RR). Respiration modulates PPG signal by respiratory-induced variations in intensity, amplitude and frequency of the PPG signal. Since the PPG signal is influenced by the respiratory cycles, it can be used for estimation of respiration rate. It is also dependent on the variations in the blood volume present in the tissues. The cardiovascular and respiratory modulations in the PPG signal are in different frequency range.

\section{A. Respiration Rate Extraction}

PPG signals are generated by the pulse oximeters that are widely used for monitoring arterial oxygen saturation. Pulse oximetry is used for continuous non-invasive monitoring of heart rate, blood pressure and respiration rate (RR). Continuous RR monitoring is useful for wellness and fitness tracking 

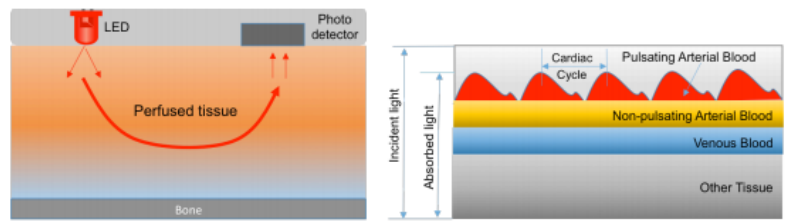

Figure 2. The fundamental principle governing reflectance-mode photoplethysmography and its relation to respiration cycles [13].

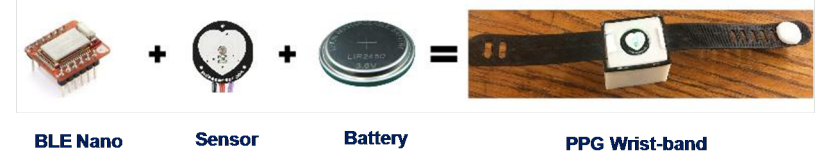

Figure 3. The systems view of the designed PPG wrist-band, the R-band.

in addition to early diagnosis of health deterioration. Several signal processing algorithms had been reported in literature for estimating the respiration rate from the PPG signals [14]. Authors in [15] studied the relation between the respiratoryinduced variations and the respiration rate. Authors in [16] used neural network with respiratory-induced features for accurate estimation of respiration rate. Authors in [17] used wavelet transform for RR estimation using respiratoryinduced variations. Authors in [18] designed, Health Chair, a system for detecting the heart and respiratory rate using sensors placed at armrests and backrests of a chair.

\section{B. Technologies for Monitoring of Respiration Rate}

It is useful to design mobile and wireless health solutions that can be used for measuring the variations in respiration (breathing) rate. Wearables are trending for inexpensive, accurate, and reliable primary diagnosis of various respiratory abnormalities such as cardiac-pulmanory disorders. Diagnosis of cardiac-pulmanory disorders is accompanied by increased respiratory rate usually more than 40 breaths per min [19]. Authors integrated a smartphone with a pulse oximeter for monitoring the breathing disorders in children [20]. The developed system presented an portable, inexpensive tool for remote screening of breathing disorders in children that performed with reasonable accuracy as compared to Polysomnography (PSG), the gold standard for such diagnose [20].

\section{MATERials \& Methods}

\section{A. R-band: A Wearable PPG System}

We designed R-band that is a reflectance-type PPG wristband. Significant portion of the wearable PPG technologies for respiration and heart rate monitoring use reflectancemode sensors. Reflectance-mode PPG has a light-emitting diode(LED) that emits light in the spectrum of infrared to near infrared region. Incident light is passed through the skin and is reflected off the subcutaneous tissue. As shown in Figure 2, in reflectance-mode PPG, a LED and a photodetector are placed in proximity to each other on the skin surface. It should be placed where there is good concentration of blood

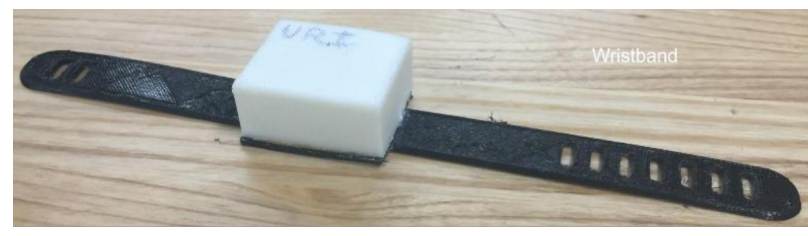

(a)

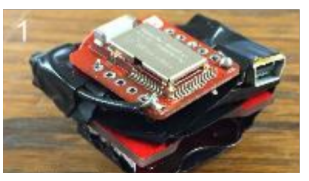

(b)

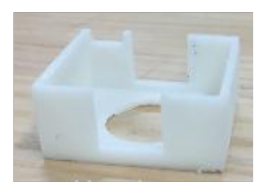

(c)
Figure 4. Showing the components of the R-band, (a) Wrist-band (b) PPG sensor with embedded Bluetooth Low Energy (BLE) (c) Housing.

vessels such as wrist. The R-band consists of 3 essential subsystems: a PPG sensor, an embedded microprocessor with a BLE radio, and a rechargeable $3.6 \mathrm{~V}$ lithium-ion coin-cell battery (see Figure 4 and Figure 3). Due to small size of sub-systems, all these can be conveniently packed within a $1.25 \times 1.25 \times 0.75$ case that was $3 \mathrm{D}$ printed for the same. Figure 4 shows the parts of the 3D printed R-band that consists of a wrist-band, a PPG sensor with embedded BLE and the Housing.

1) $P P G$ Sensor: It is a reflection-mode pulse oximeter whose principle is depicted in Figure 2. The light transmits through the skin and blood but is largely reflected by the bone and sent back to the photodetector. After the light passes through the skin, the photodetector monitors the fluctuations in light intensity that is modulated by the variations in the blood volume passing through the arteries. This allows us to extract information such as heart-rate and respiration rate from the generated PPG signal. The PPG sensor uses a green $565 \mathrm{~nm}$ LED and photodetector with a high sensitivity for the same wavelength. We choose the green light for its dominant absorption corresponding to the red blood cells. PPG sensor has an operational amplifier that has high input impedance to mitigate the electromagnetic interference. Signals were amplified before being fed to the microprocessor. Output impedance of the operational amplifier is very low that ensure that it does not load the microprocessor.

2) Signal Processing: While photodetector is the most sensitive to the wavelength of light used by the LED; it is still distorted by the unwanted wavelengths of the light present in the environment. We can reduce the amount of distortion, or noise, passed from the photodetector to the microprocessor by means of an analog low pass filter. We chose a low pass filter because the unwanted noise caused by the environment and due to the motion and leakage between the coupling of the watch and the skin are primarily at higher frequencies. Eliminating the high frequencies provided a 
better information about the blood flow. Final piece of the PPG sensor is an operational amplifier. The filtered signal is amplified by the operational amplifier, before reaching the microprocessor. Operational amplifier with high input impedance is preferred in order to avoid electromagnetic interference. This outputs an analog signal ranging from zero to three volts directly correlated with the intensity of light seen by the photodetector.

3) Data Transmission: This signal was received by the microprocessor and then sent over an available BLE connection using the standardized GATT UART Service. The signal was packed as the sets of unsigned 8-bit integers. The instance of the GATT UART Service could be updated by calling the object's function updateWriteHandler. This function was defined in the GATT UART Service header. Updating objects through its functions was the standard method used by the BLE libraries. Once the object is updated the changes were transmitted to the paired device. The PPG wrist-band was always actively sensing regardless of a connected BLE device. However, it would only update the instance of the GATT UART Service once the wrist-band was paired with a BLE device.

4) $3 D$ Printing: PPG wrist-band had two main parts, a rigid cabinet that housed its components, and an adjustable wrist-band. These two parts were fabricated at the University of Rhode Island using Fused Filament Fabrication(FFF) 3D printing. We used the Makerbot Replicator $2 x$ for our design. The adjustable wrist-band uses a thermoplastic elastomer named NinjaFlexs SemiFlex. On the other hand, the rigid case was made of Acrylonitrile Butadiene Styrene plastic. These parts were designed using the 3D CAD Design Software SOLIDWORKS. Design was planned to ensure that electronic as well as mechanical parts fit together to provide an aesthetics in R-band thus ensuring quality service to end users. The designed parts were saved in .STL format that was later fed into MakerBot MakerWare software for realizing the 3D printing. Some carefully selected tolerances were included in the design to account for the misprints and it took a few iterations of hit-and-trial to get the final finished prototype.

5) Design Profiling: The prototype was based on boxshaped container whose bottom portion was made of fusion of Acrylonitrile Butadiene Styrene slurry and mixture of Acrylonitrile Butadiene Styrene plastic dissolved in equal proportion in acetone. These parts were printed using heating plate at $110{ }^{\circ} \mathrm{C}$ and were covered with a kapton tape. The temperature of the extrude nozzle was fixed around $225^{\circ} \mathrm{C}$ during this process. Some of the failure in $3 \mathrm{D}$ printing occur when these parts does not stick fast to the build-plate. It led to wrapping of these parts and sometime complete liftingoff the plates. There were a few ways to avoid this problem. One of the solution is to use the helper-discs feature of the MakerWare software. We used a another solution for it that involved the use of a thin layered Acrylonitrile Butadiene

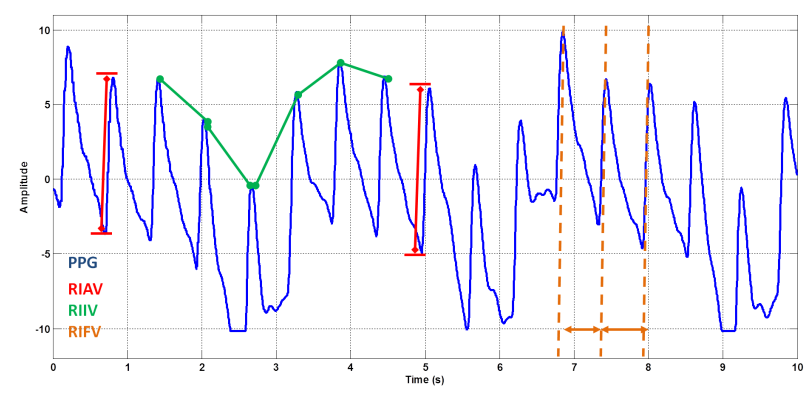

Figure 5. Respiratory-induced variations in the PPG signal. Respiratoryinduced amplitude variation (RIAV) is the change in pulse strength. Respiratory-induced intensity variation (RIIV) is the variation of perfusion baseline. Respiratory-induced frequency variation (RIFV) is the synchronization of the heart beat with the respiration rate.

Styrene slurry to the build-plate before starting the process of $3 \mathrm{D}$ printing. The flexible filaments does not need to be ultra fine for successful printing of various parts. However, having ultra fine flexible filaments can lead to a professional look of the final 3D printed prototype. For achieving the extra professional look of 3D printed prototypes, following steps could be followed. The extruder was now kept at a temperature of $215{ }^{\circ} \mathrm{C}$. It did not required a heated buildplate. However, for prototype presented in this paper, we kept the build-plate at $40{ }^{\circ} \mathrm{C}$ during this process. In this case, to ensure the adhesion of these parts to the build-plate, we needed painters tape unlike the kapton for case discussed earlier. In addition to all this, no preparation work or slurry was required in this case.

There was a possibility to get a garnished finish on the prototype with these additional steps. The Replicator $2 x$ had a dual-extruder that could be used for making a dual material and if needed a double-colored object. Sometimes the flexible filament failed due to clogging of the nuzzle that happened as a result of pushing of non-rigid filament through the nozzle by the extruder. A useful way to avoid such problem was modification of the drive block to a spring-loaded 3D printed replacement. This replacement was availed via the Thingiverse library of the Makerbot. The final part of the prototype was a clip with two prongs that was made of Acrylonitrile Butadiene Styrene. It was later used for fastening the parts of the wrist-band. The case along with the hardware needed to build R-band was tightly fixed in the wristband. The R-band was tested using a BLE scanner application such as PanoBike as it could interact with a BLE device using the GATT service.

\section{B. Feature Extraction}

As shown in Figure 5, the respiration cycle modulates the PPG signal causing three respiration-induced variations namely respiratory-induced amplitude variation (RIAV), respiratory-induced intensity variation (RIIV), and respiratory-induced frequency variation (RIFV). The respiration causes variations in heart rate (HR) so that the cardiac 
cycles are synchronized with the breathing cycles. It is known as the respiratory sinus arrhythmia (RSA). The heart rate increases during inspiration and decreases during expiration. Consequently, respiration cycle causes respiratoryinduced frequency variation (RIFV) in the PPG signal. The RIIV can be used for RR estimation regardless of the age, gender, anesthesia and the mode of ventilation. The RIIV refers to variations in the perfusion baseline that is pressure variation leading to blood exchange between the pulmonary circulation and systemic circulation. The RIAV refers to change in peripheral amplitude of the PPG signal. It is due to decrease in cardiac output resulting from reduction in ventricular filling [21]. For estimation of respiration rate, we need features that characterize the amplitude and frequencyrelated variations in the PPG signal. Usually, following two measures are being used: RSA and RIAV (amplitude variations); RIIV (frequency variations).

Figure 5 shows these measures derived from the PPG signal. We converted the PPG signals into pulses using a segmentation algorithm. Artifacts present in the PPG signal are mitigated by the segmentation algorithm to ensure accurate estimation of respiration rate. PPG signals contain DC offset that need to be suppressed before processing. We perform high pass filtering of acquired PPG signals to get DC-free PPG signal that is used for subsequent processing. We used the algorithm developed in [22] for converting the PPG signals into pulses by segmentation. This method was applied over sliding windows and could be used in realtime. After segmentation, we compute the amplitude of the pulse, minima and maxima in the intensity variations and the pulse period. The segmented PPG signal was converted into straight line segments that start from pulse initiation and go till peak value of that pulse. The outliers in the pulse periods were ignored and not used for the estimation of respiration rate. The pulse periods outside the range of $230 \mathrm{~ms}$ to $2400 \mathrm{~ms}$ were treated as outliers. These outliers were due to inevitable motion artifacts and had to be ignored for getting accurate RR estimates. Smaller windows for Fourier transform(FT) reduces the computational complexity while larger windows provide more accurate estimations. This trade-off leads us to try different windows size of 16 , 32, and 64 seconds. Finally, we used 32 seconds duration overlapping Hamming windows with 16 second skip-rate for FT estimation.

Now, we would discuss the extraction of respiratoryinduced features. RIFV is calculated using a Fourier Transform (FT) on evenly sampled intervals. FT is resampled onto an even $4 \mathrm{~Hz}$ grid using algorithm developed in [23]. We used overlapping Hamming windows of size 32 second with 16 second skip-rate. RIFV was the frequency that had maximum power within a search range of $0.067 \mathrm{~Hz}$ to 1.08 $\mathrm{Hz}$ or 4 breaths per minute to 65 breaths per minute.

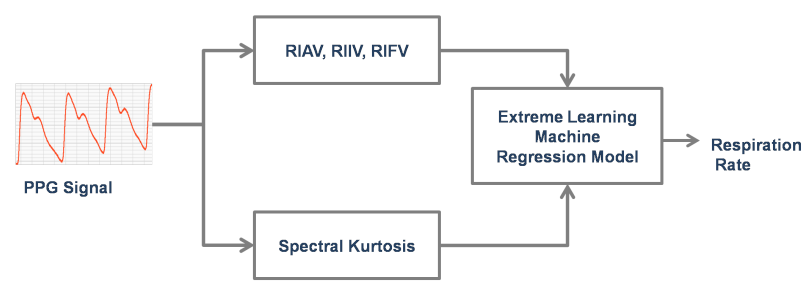

Figure 6. Proposed work flow.
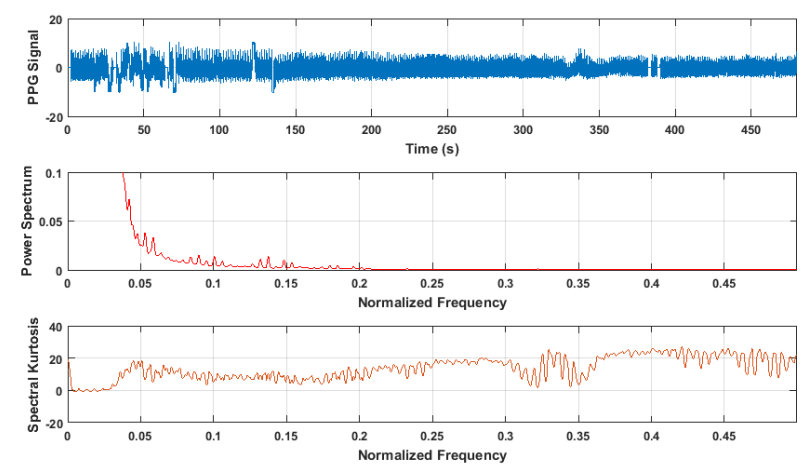

Figure 7. Spectral Kurtosis of the PPG signal sampled at $300 \mathrm{~Hz}$. We used 4096 point FFT for estimating Welch's estimate of the Spectral Kurtosis applied on time-windows of 32 second duration with 16 second overlap between the successive windows.

\section{Proposed Features: Spectral Kurtosis}

Figure 1 shows the concept of the proposed framework. The spectral kurtosis (SK) tracks the non-stationarity in a signal and detects the location of non-stationary changes in the frequency domain. Power spectrum of a signal based on Fourier transform(FT) does not track non-stationarity. Figure 7 shows the spectrum and the spectral kurtosis of a PPG signal. Author in [24] provided a procedure for estimation of spectral kurtosis of a signal. SK is robust to the presence of noise and other artifacts. These qualities of SK qualified it as a feature for tracking amplitude and frequency variations in the PPG signal. The respiration rate depends on the amplitude and frequency variations in the PPG signal. In this paper, we used a short-time Fourier transform-based estimator of SK. The SK of windowed PPG signal is used as features along with respiratoryinduced features namely RIFV, RIAV, RIIV for learning the regression between the respiration rate and the PPG signal. SK had been used for challenging detection problems [25]. Extreme Learning Machine (ELM) can learn complex nonlinear relationships such as that between the respiration rate and the features derived from the PPG signal [26]. There were three respiratory-induced variations in the PPG signal. These variations are extracted using segmentation algorithm described in [22]. These features namely RIFV, RIIV and RIAV were concatenated to spectral kurtosis of the windowed PPG signal. The feature super-set was later 
given as input to the ELM regression. The FFT was used for deriving the respiratory-induced features. Since SK is complementary to FFT, fusing these two feature set gave an improvement in accuracy of respiration rate estimation. The power spectrum of PPG signals decomposed its power as a function of frequency while the spectral kurtosis decompose the kurtosis of the spectrum as a function of frequency. Let us denote short-time Fourier transform of PPG signal by $H(\tau, f)$, then we compute kurtosis of each frequency bin for all time-windows. Mathematically, we have

$$
S K(f)=\frac{<H(\tau, f)^{4}>}{\left.<H(\tau, f)^{2}\right\rangle^{2}}-2
$$

where $\langle\cdot\rangle$ is the averaging operator. It was clear from the above equation that SK depends only on frequencies for a given PPG signal. SK captures the fourth-order statistics. SK is positive for frequency bin where signal power is higher i.e., signal is present. SK is zero for Gaussian variables. This make SK a suitable decomposition of the PPG signal that had amplitude, intensity and frequency-induced variations.

\section{Results \& Discussions}

\section{A. Data}

We used the Capnobase corpus [27], that was collected during elective surgery and routine anaesthesia. The PPG signals and capnometry data were acquired at sampling rate of $300 \mathrm{~Hz}$. The population for collection of data were 59 children (in age range of 1 to 17 years) and 35 adults (in age range of 26 to 76 years). This dataset was used in [28] containing 42 recordings of 8 minutes duration each (336 minutes in total). The ground-truth RR estimates were taken from the capnometric waveform. Each file in the corpus had signals, case name, ventilation mode and reference quantities for 8 minutes duration. Raw $\mathrm{CO} 2$ and PPG signals were also included. The labels included HR (beats per min), RR (breaths per min) and artifacts locations. The ground-truth labels were obtained from a human rater. The root mean squared error(RMSE) of proposed algorithm was found to be $(1.2 \pm 0.3$ breaths $/ \mathrm{min})$ that was an improvement over method described in [28]. The estimates of respiration rate were obtained for time-windows of 32 second duration of the PPG signal. Windows with motion artifacts (outliers) were ignored.

Training, development and evaluation sets are obtained by making disjoint sub-sets of Capnobase data [?]. The $50 \%$ data was chosen as training set, $10 \%$ as development set and $40 \%$ for evaluation. SK-ELM based estimator was trained on first subset. Development set was used for tuning the various parameters and finding the optimized parameter values. Finally, we test the method on evaluation dataset. PPG signal was first preprocessed with a fifth-order Butterworth filter with lower and upper cut-off frequencies at $0.08 \mathrm{~Hz}$ and 3 $\mathrm{HZ}$ respectively. Capnobase data used for our studies was obtained from 29 children and 13 adults. Next, the region of signal (ROS) was used to extract RIFV, RIIV, RIAV and SK features. In this paper, we used 1024 point Welch's estimate of SK. These features were fed to the ELM regression model with 2048 nodes in hidden layer (see Figure 6). Radial basis function $(\mathrm{RBF})$ was found to be the best non-linearity for hidden layer with respect to our regression problem. We used the capnometry as ground-truth respiration rate (reference labels). The root mean squared error (RMSE) in breaths per minute over $N$ time-windows was calculated as

$$
\epsilon_{R R}=\sqrt[2]{\frac{1}{N} \sum_{i=1}^{N}|R R[i]-\hat{R R}[i]|^{2}}
$$

where $R R[i]$ and $\hat{R R}[i]$ were the ground-truth and estimated RR values for $i$-th time-window, respectively. The groundtruth RR values were included in the data-set provided by authors in [29]. Using proposed method, we could get improved accuracy of 1.2 breaths/min (root mean squared error).

\section{CONCLUSION}

We designed a wearable PPG system named R-band (Respiration band) for acquisition of the PPG signals. We proposed a method that uses spectral kurtosis features along with the state-of-the-art respiratory-induced frequency, intensity and amplitude features. Spectral kurtosis(SK) features track the non-stationary components in the photoplethysmography (PPG) signal unlike the Fourier transform (FT) that captures only the stationary components. Respiratory-induced features were derived from FT. We fused the SK with respiratory-induced features thus combining the complementary signatures in PPG signals that led to improved accuracy. The fused feature set was used for training an Extreme Learning Machine (ELM) regression model for accurate estimation of the respiration rate. Capnobase data was used for benchmarking the proposed algorithms.

\section{REFERENCES}

[1] U. National Instite of Health, "Disease Statistics," http://www.nhlbi.nih.gov/about/documents/factbook/2012/chapter4, 2012, [Online; accessed 02-April-2016].

[2] D. Mozaffarian, E. J. Benjamin, A. S. Go, D. K. Arnett, M. J. Blaha, M. Cushman, S. R. Das, S. de Ferranti, J.-P. Després, H. J. Fullerton et al., "Heart disease and stroke statistics2016 update a report from the american heart association," Circulation, pp. CIR-0 $000000000000350,2015$.

[3] V. Mohsenin, "Sleep-related breathing disorders and risk of stroke," Stroke, vol. 32, no. 6, pp. 1271-1278, 2001.

[4] H. Dubey, M. R. Mehl, and K. Mankodiya, "Bigear: Inferring the ambient and emotional correlates from smartphonebased acoustic big data," in 2016 IEEE First International Conference on Connected Health: Applications, Systems and Engineering Technologies (CHASE), June 2016, pp. 78-83. 
[5] H. Dubey, J. C. Goldberg, M. Abtahi, L. Mahler, and K. Mankodiya, "Echowear: Smartwatch technology for voice and speech treatments of patients with parkinson's disease," in Proceedings of the Conference on Wireless Health, ser. WH '15. New York, NY, USA: ACM, 2015, pp. 15:1-15:8.

[6] H. Dubey, J. Yang, N. Constant, A. M. Amiri, Q. Yang, and K. Makodiya, "Fog data: Enhancing telehealth big data through fog computing," in The Fifth ASE International Conference on Big Data, ACM BigData 2015, Kaohsiung, Taiwan. ACM, 2015.

[7] M. E. Kunik, K. Roundy, C. Veazey, J. Souchek, P. Richardson, N. P. Wray, and M. A. Stanley, "Surprisingly high prevalence of anxiety and depression in chronic breathing disorders," Chest Journal, vol. 127, no. 4, pp. 1205-1211, 2005.

[8] T. Wardlaw, E. Johansson, and M. Hodge, "Pneumonia: the forgotten killer of children. new york, usa/geneva, ch: The united nations childrens fund (unicef)," World Health Organization (WHO), 2006.

[9] H. Dubey, R. Kumaresan, and K. Mankodiya, "Harmonic sum-based method for heart rate estimation using ppg signals affected with motion artifacts," Journal of Ambient Intelligence and Humanized Computing, pp. 1-14, 2016.

[10] S. Quan, J. C. Gillin, M. Littner, and J. Shepard, "Sleeprelated breathing disorders in adults: Recommendations for syndrome definition and measurement techniques in clinical research. editorials," Sleep, vol. 22, no. 5, pp. 662-689, 1999.

[11] W. Karlen, H. Gan, M. Chiu, D. Dunsmuir, G. Zhou, G. A. Dumont, and J. M. Ansermino, "Improving the accuracy and efficiency of respiratory rate measurements in children using mobile devices," PloS one, vol. 9, no. 6, p. e99266, 2014.

[12] P. B. Lovett, J. M. Buchwald, K. Stürmann, and P. Bijur, "The vexatious vital: neither clinical measurements by nurses nor an electronic monitor provides accurate measurements of respiratory rate in triage," Annals of emergency medicine, vol. 45, no. 1, pp. 68-76, 2005.

[13] J. G. Webster, Design of pulse oximeters. CRC Press, 1997.

[14] K. Nakajima, T. Tamura, T. Ohta, H. Miike, and P. Öberg, "Photoplethysmographic measurement of heart and respiratory rates using digital filters," in Engineering in Medicine and Biology Society, 1993. Proceedings of the 15th Annual International Conference of the IEEE. IEEE, 1993, pp. 1006-1007.

[15] J. Li, J. Jin, X. Chen, W. Sun, and P. Guo, "Comparison of respiratory-induced variations in photoplethysmographic signals," Physiological measurement, vol. 31, no. 3, p. 415, 2010.

[16] A. Johansson, "Neural network for photoplethysmographic respiratory rate monitoring," Medical and Biological Engineering and Computing, vol. 41, no. 3, pp. 242-248, 2003.

[17] P. S. Addison, J. N. Watson, M. L. Mestek, and R. S. Mecca, "Developing an algorithm for pulse oximetry derived respiratory rate (rroxi): a healthy volunteer study," Journal of clinical monitoring and computing, vol. 26, no. 1, pp. 45-51, 2012.
[18] E. Griffiths, T. S. Saponas, and A. Brush, "Health chair: implicitly sensing heart and respiratory rate," in Proceedings of the 2014 ACM International Joint Conference on Pervasive and Ubiquitous Computing. ACM, 2014, pp. 661-671.

[19] J. P. Metlay, W. N. Kapoor, and M. J. Fine, "Does this patient have community-acquired pneumonia?: Diagnosing pneumonia by history and physical examination," Jama, vol. 278, no. 17, pp. 1440-1445, 1997.

[20] A. Garde, P. Dehkordi, W. Karlen, D. Wensley, J. M. Ansermino, and G. A. Dumont, "Development of a screening tool for sleep disordered breathing in children using the phone oximeter," PloS one, vol. 9, no. 11, p. e112959, 2014.

[21] A. J. Buda, M. R. Pinsky, N. B. Ingels Jr, G. T. Daughters, E. B. Stinson, and E. L. Alderman, "Effect of intrathoracic pressure on left ventricular performance," New England Journal of Medicine, vol. 301, no. 9, pp. 453-459, 1979.

[22] W. Karlen, J. M. Ansermino, and G. Dumont, “Adaptive pulse segmentation and artifact detection in photoplethysmography for mobile applications." in Conference proceedings:... Annual International Conference of the IEEE Engineering in Medicine and Biology Society. IEEE Engineering in Medicine and Biology Society. Annual Conference, vol. 2012, 2011, pp. 3131-3134.

[23] R. D. Berger, S. Akselrod, D. Gordon, and R. J. Cohen, "An efficient algorithm for spectral analysis of heart rate variability," Biomedical Engineering, IEEE Transactions on, no. 9, pp. 900-904, 1986.

[24] J. Antoni, "The spectral kurtosis: a useful tool for characterising non-stationary signals," Mechanical Systems and Signal Processing, vol. 20, no. 2, pp. 282-307, 2006.

[25] J. Antoni and R. Randall, "The spectral kurtosis: application to the vibratory surveillance and diagnostics of rotating machines," Mechanical Systems and Signal Processing, vol. 20, no. 2, pp. 308-331, 2006.

[26] G.-B. Huang, Q.-Y. Zhu, and C.-K. Siew, "Extreme learning machine: theory and applications," Neurocomputing, vol. 70, no. 1 , pp. 489-501, 2006.

[27] http://www.capnobase.org/index.php?id=857.

[28] W. Karlen, A. Garde, D. Myers, C. Scheffer, J. M. Ansermino, G. Dumont et al., "Respiratory rate assessment from photoplethysmographic imaging," in Engineering in Medicine and Biology Society (EMBC), 2014 36th Annual International Conference of the IEEE. IEEE, 2014, pp. 5397-5400.

[29] W. Karlen, S. Raman, J. M. Ansermino, G. Dumont et al., "Multiparameter respiratory rate estimation from the photoplethysmogram," Biomedical Engineering, IEEE Transactions on, vol. 60, no. 7, pp. 1946-1953, 2013. 